\title{
Suppression of Alzheimer's disease-related phenotypes by the heat shock protein 70 inducer, geranylgeranylacetone, in APP/PS1 transgenic mice via the ERK/p38 MAPK signaling pathway
}

\author{
YUAN SUN* , JIANG-RONG ZHANG* and SHUYAN CHEN
}

Department of Geratology, Xinhua Hospital, Shanghai Jiaotong University School of Medicine, Shanghai 200092, P.R. China

Received January 7, 2016; Accepted January 6, 2017

DOI: $10.3892 /$ etm.2017.5253

\begin{abstract}
HSP70 overexpression has a remedying effect in neurodegenerative diseases. In Alzheimer's disease (AD), the suppressive effects of HSP70 overexpression on AD-related phenotypes and the underlying mechanisms are unknown. In the current study, the effect of geranylgeranylacetone (GGA), a non-toxic inducer of heat shock protein (HSP)-70 expression, on cognitive function and other pathological phenotypes were evaluated in APP/PS1 mice. It was observed that all doses of orally administered GGA (200, 400, and $800 \mathrm{mg} / \mathrm{kg} /$ day $)$ improved cognitive deficit $(\mathrm{P}<0.05)$ and lowered the levels of amyloid- $\beta(\mathrm{A} \beta)$ peptide $(\mathrm{P}<0.05)$ in $\mathrm{APP} / \mathrm{PS} 1$ mice. GGA treatment also increased the levels of low density lipoprotein receptor-related protein 1 (LRP-1) $(\mathrm{P}<0.05)$, while the levels of p-glycoprotein and receptor for advanced glycation end products were unaltered. Significant decreases in the levels of inflammatory cytokines, namely tumor necrosis factor- $\alpha$, interleukin-1 $\beta$ and cyclooxygenase-2, were also observed in the GGA-treated mice $(\mathrm{P}<0.05)$. Subsequent treatment with the HSP70 inhibitor quercetin caused significant decreases in the levels of phosphorylated (p)-p38 mitogen-activated protein kinase (p38 MAPK) and p-extracellular signal-regulated protein kinases $(\mathrm{ERK} ; \mathrm{P}<0.05)$, indicating that ERK/p38 MAPK signaling in AD-related phenotypes may be suppressed by oral administration of GGA. Finally, in APP/PS1 mice treated with GGA+SB-203580 (p38 inhibitor) and GGA+PD98059 (ERK inhibitor), it was observed that orally administered GGA led to the activation of ERK/p38 MAPK signaling $(\mathrm{P}<0.05)$ and increased LRP-1 expression
\end{abstract}

Correspondence to: Dr Shuyan Chen, Department of Geratology, Xinhua Hospital, Shanghai Jiaotong University School of Medicine, 1665 KongJiang Road, Shanghai 200092, P.R. China

E-mail: sychen_xh@126.com

*Contributed equally

Key words: Alzheimer's disease, heat shock protein 70, geranylgeranylacetone, amyloid- $\beta$, extracellular signal-regulated kinase/p38 mitogen-activated protein kinase pathway
$(\mathrm{P}<0.05)$, which subsequently aided the clearance of $\mathrm{A} \beta 40$ and $\mathrm{A} \beta 42(\mathrm{P}<0.05)$ and alleviated AD-related phenotypes. These results indicate that oral administration of GGA in APP/PS1 mice alleviates $A D$-related phenotypes by regulation of the ERK/p38 MAPK signaling pathway. Thus, GGA may be a potential therapeutic for the treatment of AD.

\section{Introduction}

Alzheimer's disease (AD) is the most prevalent form of dementia and is defined as a neurodegenerative disorder that occurs in the elderly (especially those aged $>60$ years). AD is the fourth most common cause of human mortality after cardiovascular disease, cancer and stroke, and its incidence rate is increasing due to an aging population worldwide. Thus, the disease is a considerable social economic burden for families and society $(1,2)$. Clinically, AD is characterized by a range of neurological symptoms, including memory deficit, cognitive impairment and movement disorder (3). Pathologically, the hallmark of $\mathrm{AD}$ is an accumulation of insoluble amyloid- $\beta(\mathrm{A} \beta)$ deposits within amyloid plaques, mainly consisting of $\mathrm{A} \beta 40$ and $A \beta 42$ peptides $(4,5)$. Deposition of $A \beta$ is possibly related to an imbalance between production and clearance of $A \beta$, with defective clearance considered to be the leading cause (6). In particular, $A \beta$-rich oligomers, protofibrils and fibrils, formed by the self-assembly of $A \beta$, are potentially responsible for the neurotoxic effects of $A \beta$ in the pathogenesis of $A D(7)$.

Previous studies have suggested different mechanisms for the removal of $\mathrm{A} \beta$ from the brain $(8,9)$. Receptor-mediated transport of $\mathrm{A} \beta$ across the blood brain barrier (BBB) to the periphery is considered to be a key mechanism for $A \beta$ removal, involving low-density lipoprotein receptor-related protein (LRP), the receptor for advanced glycation end products (RAGE) and the adenosine triphosphate-binding cassette transporter p-glycoprotein (p-gp) (10). Therefore, identifying cellular factors that affect $A \beta$ production and clearance aid in the development of novel therapeutics for the treatment of AD. In addition, studies over the past decade have suggested a key role of inflammation in the pathogenesis of AD (11).

Results of past studies have suggested that AD is a 'protein misfolding disorder', characterized by misfolded and aggregated proteins in the brain of AD patients $(12,13)$. In healthy individuals, this is typically prevented by heat shock proteins (HSPs) acting as molecular chaperones. These integrate with 
non-native proteins to facilitate their folding into a native state, thus preventing misfolding and aggregation, particularly under cell stress $(14,15)$. However, a potential link between HSPs and AD is not well understood. A number of past studies have suggested a potential role of HSPs in AD pathogenesis, particularly for HSP70 (16-18). In addition, neuroprotective activities of HSP70 regarding the anti-aggregation and initiation of $A \beta$ clearance have been reported (19), suggesting that inducers of HSP70 may be potential therapeutic targets in the treatment of AD.

The present study aimed to determine how raised levels of HSP70 alleviate AD-related phenotypes in the pathogenesis of AD. In particular, the effects of the non-toxic HSP-inducer geranylgeranylacetone (GGA) (20) on AD phenotypes were investigated via Y-maze, object recognition, and Morris water maze tests in APP/PS1 mice. The pathological characteristics related to these phenotypes were also evaluated through western blot analysis and ELISA.

\section{Materials and methods}

Animals. Three-month-old male APP/PS1 double transgenic mice [B6.Cg-Tg(APPswe,PSEN1dE9)85Dbo/Mmjax] $(n=112)$, each weighing 25-35 g, were obtained from Jackson Laboratory (Bar Harbor, ME, USA) and 3-month-old littermate male wild-type (WT) mice $(n=24)$, each weighing 25-35 g, also purchased from Jackson Laboratory, were used as controls. The Tg mice express a chimeric mouse/human amyloid precursor protein (APP695swe) and a mutant human presenilin 1 protein (PSEN1dE9) with an exon 9 deletion, both under the control of murine prion promoter elements (21). Mice were kept under a 12 -h light-dark cycle at $23 \pm 1^{\circ} \mathrm{C}$ and $50-60 \%$ humidity with water and food available ad libitum. For oral administration of GGA, APP/PS1 and WT mice were administered GGA-supplemented chow from the age of 3 to 12 months ( 9 months of administration period), according to a previous method (22). Mice were divided into the following six groups ( $\mathrm{n}=8$ per group): WT, Tg (APP/PS1 mice, model of AD), Tg+GGA200 (oral administration of GGA 200 mg/kg/day), Tg+GGA400 (oral administration of GGA 400 mg/kg/day), Tg+GGA800 (oral administration of GGA $800 \mathrm{mg} / \mathrm{kg} /$ day) and WT+GGA800 (oral administration of GGA $800 \mathrm{mg} / \mathrm{kg} / \mathrm{day}$ ). To investigate whether GGA affects MAPK signaling in APP/PS1 mice, mice were divided into five groups $(\mathrm{n}=8)$ : WT, $\mathrm{Tg}, \mathrm{Tg}+\mathrm{GGA} 400$, $\mathrm{Tg}+\mathrm{GGA}+$ quercetin (intraperitoneal injection of $100 \mathrm{mg} / \mathrm{kg}$ quercetin 3 times/week for 6 months, HSP70 inhibitor) and WT+GGA800. Furthermore, mice (WT and APP/PS1) were also divided into six groups $(\mathrm{n}=8)$ : WT, Tg, Tg+GGA400, $\mathrm{Tg}+\mathrm{GGA}+$ quercetin, $\mathrm{Tg}+\mathrm{GGA}+\mathrm{SB}-203580$ (p38 inhibitor, intracerebroventricular injection of SB-203580 $10 \mathrm{nmol} /$ day for 9 months); Tg+GGA+PD98059 (ERK inhibitor, intracerebroventricular injection of PD98059 $10 \mathrm{nmol}$ for 9 months). The animal protocol was approved by the Ethics of Animal Experiments Committee at Jiaotong University School of Medicine (Shanghai, China).

Morris water maze (MWM) test. An MWM test was performed in order to evaluate memory function, as previously described (23). Briefly, a circular water tank (100 cm diameter,
$40 \mathrm{~cm}$ height) was divided into quadrants and filled with water $\left(23 \pm 1^{\circ} \mathrm{C}\right)$ to a depth of $15.5 \mathrm{~cm}$. The water was made opaque by the addition of white tempera powder to the pool. A transparent escape platform (10 cm diameter, $20 \mathrm{~cm}$ height) was hidden $1 \mathrm{~cm}$ below the water surface and positioned at the midpoint of one quadrant. Each mouse received daily training for six days using a single hidden platform in successive quadrants. Latency to escape from the water maze (finding the submerged platform) was recorded for each trial. On day seven, mice were subjected to a probe test in which the platform was removed and each mouse was permitted to swim freely for $60 \mathrm{sec}$. Data were recorded using SMART 2.5 video tracking software (Harvard Apparatus, Holliston, MA, USA).

Y-maze spontaneous alternation test. A Y-maze spontaneous alternation test was performed in order to evaluate recognition memory, as described previously (24). The Y-maze was made of black-painted wood and each arm of the maze was $50 \times 10 \times 20 \mathrm{~cm}$. Each mouse was released in the middle of the apparatus and permitted to move through the maze for $8 \mathrm{~min}$. The series of arm entries were visually observed. Spontaneous alteration was defined as successive entries into each of the three arms in overlapping triplet sets. Alteration behavior (\%) was calculated as the ratio of the number of alternations to the total number of arm entries.

Object recognition test. An object recognition test was carried out as a second measure of recognition memory, as described previously (25). Briefly, mice were kept in a test chamber $(25 \times 25 \times 40 \mathrm{~cm})$ overnight under a $12-\mathrm{h}$ light-dark cycle at controlled temperature $\left(23 \pm 1^{\circ} \mathrm{C}\right)$ and humidity (50-60\%) with water and food ad libitum, prior to training. During training, two identical objects (round filter units, $33 \mathrm{~mm}$ diameter, $27 \mathrm{~mm}$ height) were placed in the chamber and mice were permitted to explore for $10 \mathrm{~min}$. The following day, one of the objects was replaced with a new object (plastic cone, $25 \mathrm{~mm}$ diameter, $30 \mathrm{~mm}$ height). The object recognition index was defined as the percentage of time spent sniffing or touching the novel object with the nose in a 5 min period. All training and test trials were video recorded and analyzed using EthoVision XT8.5 (Noldus Information Technology, Wageningen, Netherlands).

Sandwich ELISA (sELISA) of $A \beta$. Levels of $\mathrm{A} \beta 40$ and $\mathrm{A} \beta 42$ peptides in mouse brain tissue from all groups were determined as described previously (26). The mice were anaesthetized by intraperitoneal injection with $1 \%$ pentobarbital sodium $(50 \mathrm{mg} / \mathrm{kg}$, Sumitomo Dainippon Pharmaceutical Co., Ltd, Osaka, Japan) and decapitated to obtain brain tissues. Briefly, brain hemispheres were homogenized in $50 \mathrm{mM}$ Tris-hydrochloride $(\mathrm{HCl})$ buffer $(\mathrm{pH} 7.6)$ supplemented with $150 \mathrm{mM}$ sodium chloride, then centrifuged. A total of $0.5 \mathrm{M}$ guanidine- $\mathrm{HCl}$ was added to the supernatants (soluble fractions), while precipitates were solubilized by sonication in $6 \mathrm{M}$ guanidine- $\mathrm{HCl}$, then centrifuged at $20,000 \mathrm{x}$ g for $1 \mathrm{~h}$ at $4^{\circ} \mathrm{C}$ to remove insoluble material. The quantity of $A \beta 40$ and $A \beta 42$ in each fraction was determined by sELISA, according to the manufacturer's protocol (cat. no. 27721; Immuno-Biological Laboratories, Inc., Minneapolis, MN, USA). The experiments were performed 3 times. 
Immunoblotting. Whole proteins were prepared from the brain tissues of all 12-month-old WT and APP/PS1 mice in $200 \mu \mathrm{l}$ lysis buffer (30 mM Tris-HCl pH 8.0, $150 \mathrm{mM} \mathrm{NaCl}, 1 \%$ NP-40, $1 \mathrm{mM}$ phenylmethylsulfonyl fluoride and protease inhibitor cocktail; all from Sigma-Aldrich; Merck KGaA, Darmstadt, Germany), and total protein concentration was determined with a Bio-Rad Protein Assay kit (Bio-Rad Laboratories, Inc., Hercules, CA, USA). Then samples (50 $\mu$ g protein) were added to $10 \%$ SDS-PAGE gels at a ratio of 1:4, boiled for $5 \mathrm{~min}$, and transferred for $2 \mathrm{~h}$ to PVDF membranes. After blocking for $1 \mathrm{~h}$ with $5 \%$ non-fat dry milk at room temperature in PBS, membranes were incubated overnight at room temperature with the following primary antibodies: ERK (cat. no. 9102; Cell Signaling Technology, Inc., Danvers, MA, USA), p-ERK (cat. no. 9101; Cell Signaling Technology, Inc.), p38 (cat. no. sc-7972; Santa Cruz Biotechnology, Inc., Dallas, TX, USA), p-p38 (cat. no. sc-7973; Santa Cruz Biotechnology, Inc.), JNK (cat. no. sc-137019; Santa Cruz Biotechnology, Inc.), p-JNK (cat. no. sc-293136; Santa Cruz Biotechnology, Inc.), LRP1 (cat. no. sc-16168; Santa Cruz Biotechnology, Inc.), RAGE (cat. no. sc-8230; Santa Cruz Biotechnology, Inc.), p-gp (cat. no. sc-13131; Santa Cruz Biotechnology, Inc.) at a dilution of 1:2,000. Membranes were then incubated for $45 \mathrm{~min}$ with goat anti-rabbit horseradish peroxidase-coupled secondary antibodies (cat. no. sc-3836; Santa Cruz Biotechnology, Inc.; 1:3,000) at room temperature. The bound antibodies were detected using an enhanced chemiluminescence detection kit (Thermo Fisher Scientific, Inc., Waltham, MA, USA). For gel loading control, membranes were re-probed with a monoclonal $\alpha$-tubulin antibody (cat. no. T5168; 1:1,000; Sigma-Aldrich; Merck KGaA). The band densities were quantified with Image Pro Plus 4.5 software (Media Cybernetics, Inc., Rockville, MD, USA). The experiments were performed 3 times.

Statistical analysis. Data are presented as the mean \pm standard error of the mean, and were analyzed using SPSS 16.0 software (SPSS, Inc., Chicago, IL, USA). One or two way analysis of variance followed by a Tukey test was performed to assess differences between $>$ three groups. A Student's t-test for unpaired results was performed to evaluate differences between two groups. $\mathrm{P}<0.05$ was considered to indicate a statistically significant difference.

\section{Results}

Orally administered GGA improves cognitive function in APP/PS1 mice. AD-related phenotypes become apparent in 12-month-old APP/PS1 mice, therefore APP/PS1 and wild-type mice were orally administered GGA from the age of 3 to 12 months ( 9 months of administration period), according to a previous method (22). Y-maze and object recognition tests were then performed to examine potential dose-dependent effects of GGA on spatial learning and memory in APP/PS1 mice at the end of the administration period. Results of the Y-maze demonstrated that the alternation rates of APP/PS1 mice were significantly lower than those of WT mice $(\mathrm{P}<0.05)$, and that GGA administration (400 and $800 \mathrm{mg} / \mathrm{kg} /$ day) significantly increased the alternation rates of APP/PS1 mice, relative to untreated APP/PS1 mice ( $\mathrm{P}<0.05$; Fig. 1A). Similarly, in the object recognition test, APP/PS1 mice exhibited a significantly lower exploratory preference for a novel object, compared with WT mice, and all doses of orally administered GGA (200-800 mg/kg/day) significantly increased the exploratory preference of APP/PS1 mice, relative to untreated APP/PS1 mice $(\mathrm{P}<0.05$; Fig. 1B).

An MWM test was also performed to evaluate spatial memory in APP/PS1 mice. Mice were trained daily for six days and the time taken to reach a hidden platform (escape latency) was recorded. As presented in Fig. 1C, APP/PS1 mice exhibited a significantly longer escape latency, compared with WT mice $(\mathrm{P}<0.05)$, and all doses of GGA significantly reduced the escape latency in APP/PS1 mice, relative to untreated APP/PS1 mice $(\mathrm{P}<0.05)$. In addition, the significantly lower platform cross frequencies in APP/PS1 mice, relative to WT mice $(\mathrm{P}<0.05)$, were significantly increased by all doses of GGA, relative to untreated APP/PS1 mice $(\mathrm{P}<0.05$; Fig. 1D). However, oral administration of GGA did not affect spatial learning and memory in WT mice (Fig. 1A-D). These data suggest that oral administration of GGA (200-800 mg/kg/day) causes significant alleviation of cognitive deficit in APP/PS1 mice.

Orally administered GGA reduces A $\beta$ levels in APP/PS1 mice. Based on the cytoprotective activity of HSP70 overexpression against $A \beta$ neurotoxicity in mice (19), the present study evaluated the effects of HSP70 inducer GGA on the levels of A $\beta$ in APP/PS1 mice by ELISA. As presented in Fig. 2A and B, the levels of soluble $A \beta 40$ and $A \beta 42$ did not differ significantly between WT mice and GGA-administered WT mice, while $\mathrm{A} \beta 40$ and $\mathrm{A} \beta 42$ levels were significantly higher in APP/PS1 mice, relative to untreated WT mice $(\mathrm{P}<0.05)$. In turn, oral administration of GGA (400 and $800 \mathrm{mg} / \mathrm{kg} / \mathrm{day}$ ) significantly reversed the elevated levels of soluble $A \beta 40$ and $A \beta 42$ in the brain of APP/PS1 mice $(\mathrm{P}<0.05)$, suggesting that oral administration of GGA may lower A $\beta$ levels in APP/PS1 mice.

Orally administered GGA may stimulate $A \beta$ clearance in $A P P / P S 1$ mice. As a removal mechanism of $\mathrm{A} \beta$ from the brain is receptor-mediated transport of $A \beta$ across the $B B B$ into the periphery, the current study evaluated the effect of orally administered GGA on the level of proteins involved in receptor-mediated $\mathrm{A} \beta$ elimination in APP/PS1 mice, namely LRP-1, RAGE and p-gp, by western blot analysis. As depicted in Fig. 3A, the level of LRP-1 was significantly lower in the brain of APP/PS1 mice relative to WT mice $(\mathrm{P}<0.05)$, and all doses of orally administered GGA significantly reversed the lower levels of LRP-1 in APP/PS1 mice $(\mathrm{P}<0.05)$. However, the significantly increased levels of RAGE and lower levels of p-gp in APP/PS1 mice, compared with WT mice $(\mathrm{P}<0.05)$, were not altered by GGA administration (Fig. 3B and C). These results, suggest that oral administration of GGA may in part stimulate $A \beta$ clearance through upregulation of LRP-1.

Orally administered GGA on inflammation in the brain of APP/PS1 mice. Based on the role of inflammation in AD pathogenesis, the current study evaluated the effect of GGA on the levels of inflammatory mediators in APP/PS1 mice by western blotting (Fig. 4). Relative to WT mice, APP/PS1 mice exhibited significantly higher levels of TNF- $\alpha$, IL-1 $\beta$ and COX-2 $(\mathrm{P}<0.05)$. In turn, all doses of orally administered GGA significantly reversed the elevated levels of TNF- $\alpha$, 
A
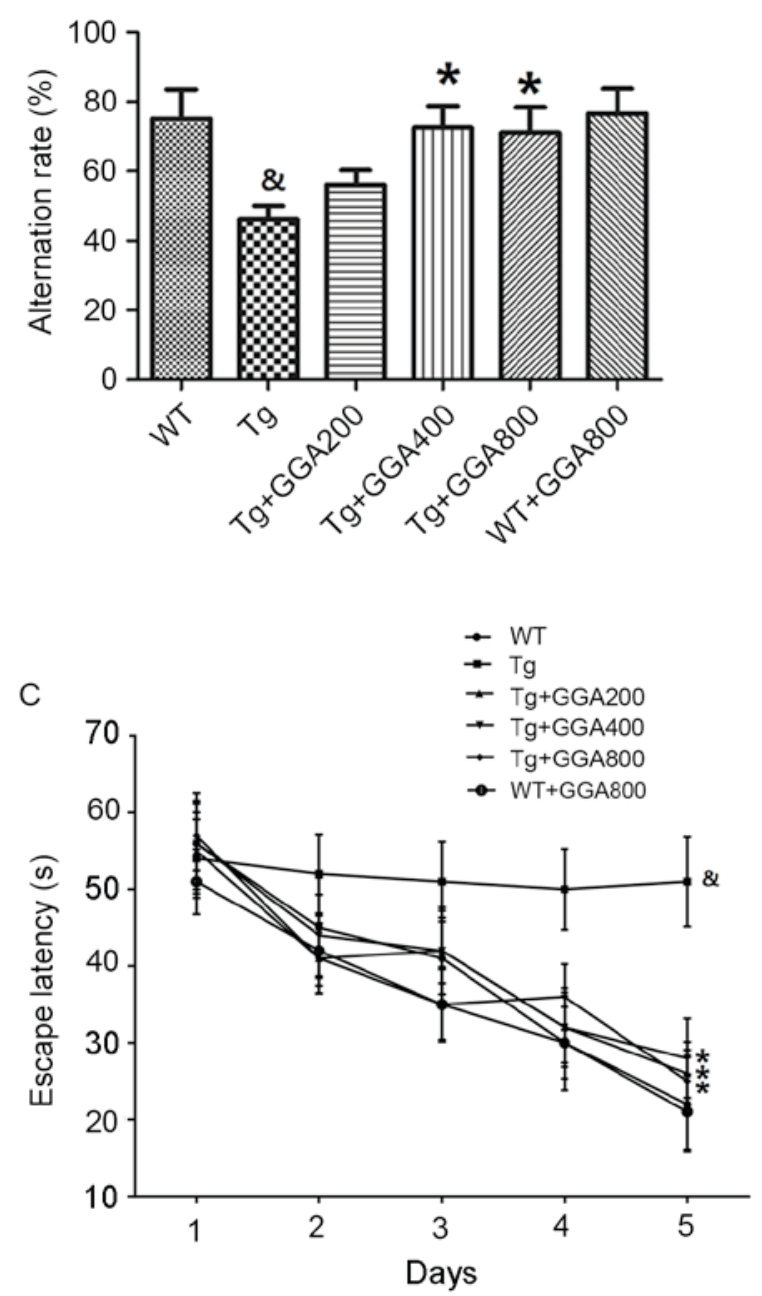

B

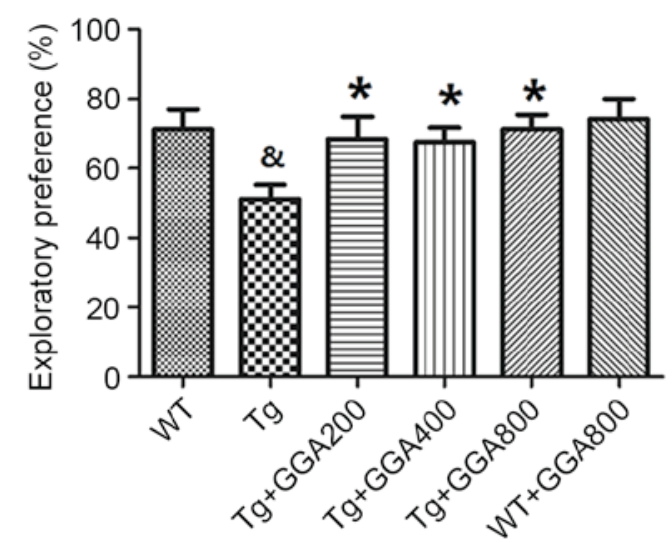

$\mathrm{D}$

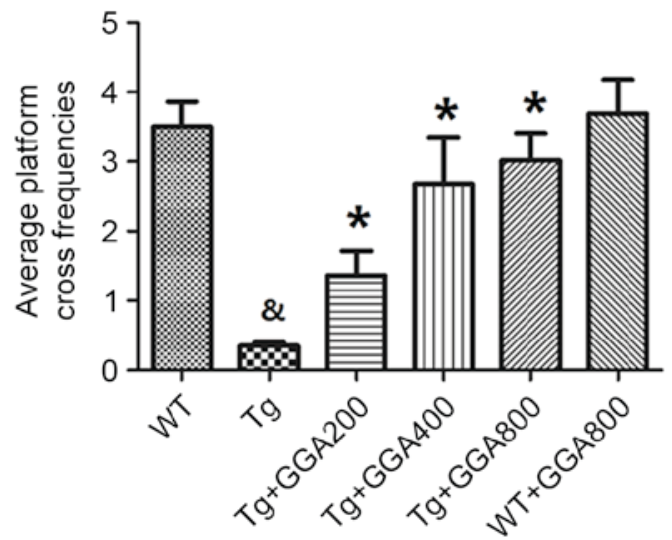

Figure 1. Effect of orally administered GGA on cognitive function in APP/PS1 mice. (A) Y-maze, (B) object recognition and (C) MWM tests were conducted in 12-month-old WT and APP/PS1 mice administered with GGA between the ages of 3-12 months. (D) Platform cross frequencies in the MWM were also measured. ${ }^{\circledR} \mathrm{P}<0.05$ vs. untreated WT mice, ${ }^{*} \mathrm{P}<0.05$ vs. untreated Tg mice. GGA, geranylgeranylacetone; APP/PS1, transgenic mouse strain; MWM, Morris water maze; WT, wild type; Tg, transgenic.
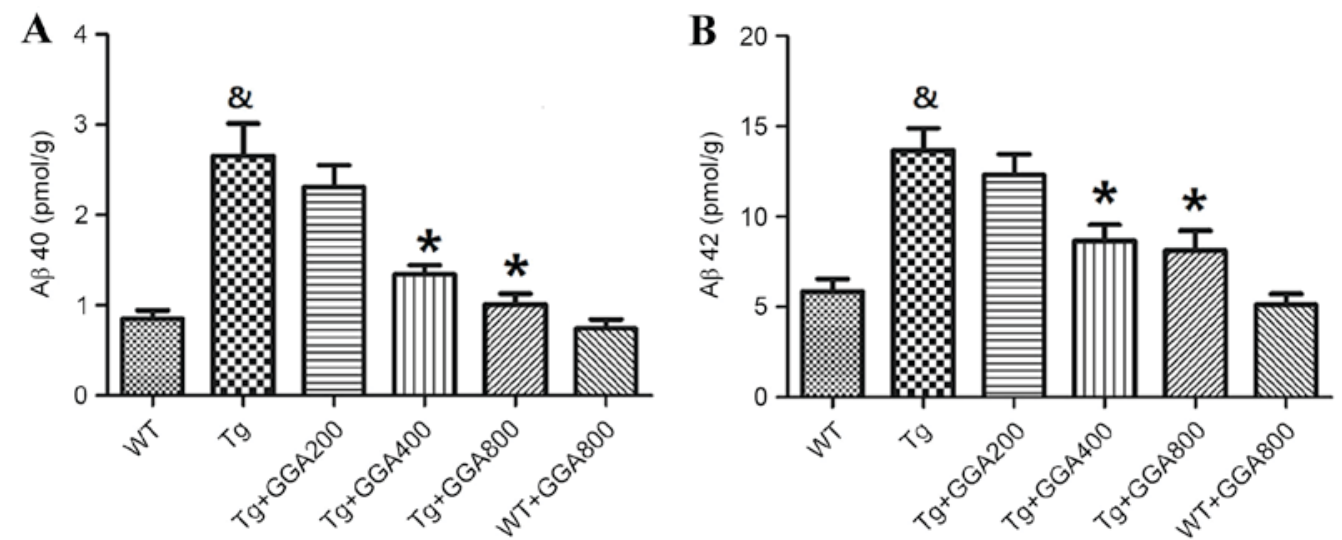

Figure 2. Effect of orally administered GGA on A $\beta$ levels in APP/PS1 mice. Levels of soluble (A) A $\beta 40$ and (B) A $\beta 42$ in the brain of WT and APP/PS1 mice administered with GGA were measured by sandwich ELISA. ${ }^{\&} \mathrm{P}<0.05$ vs. untreated WT mice, ${ }^{*} \mathrm{P}<0.05$ vs. untreated Tg mice. GGA, geranylgeranylacetone; $\mathrm{A} \beta$, amyloid- $\beta ; \mathrm{APP} / \mathrm{PS} 1$, transgenic mouse strain; WT, wild type; Tg, transgenic.

IL-1 $\beta$ and COX-2 in the brain of APP/PS1 mice $(\mathrm{P}<0.05)$. By contrast, GGA administration had no effect on the levels of TNF- $\alpha$, IL-1 $\beta$ and COX-2 in WT mice.
Underlying mechanism of GGA effects on AD-related phenotypes. As MAPK is considered to serve a key role in $\mathrm{AD}$ pathophysiology (27), the current study investigated whether 

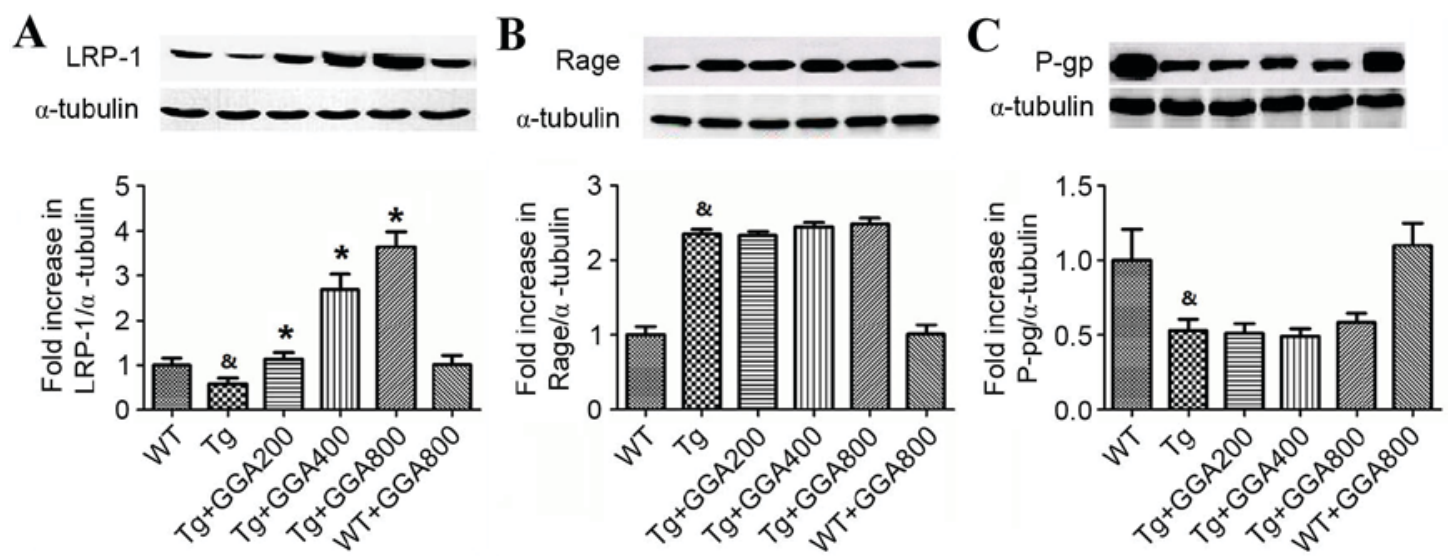

Figure 3. Effect of orally administered GGA on the levels of A $\beta$ clearance proteins in APP/PS1 mice. Whole cell extracts were prepared from the brains of GGA-treated WT and APP/PS1 mice prior to western blot analysis to measure the levels of (A) LRP-1, (B) RAGE and (C) p-gp expression. $\alpha$-Tubulin was used as an internal standard. ${ }^{\&} \mathrm{P}<0.05$ vs. untreated WT mice, ${ }^{*} \mathrm{P}<0.05$ vs. untreated Tg mice. GGA, geranylgeranylacetone; $\mathrm{A} \beta$, amyloid- $\beta$; APP/PS1, transgenic mouse strain; WT, wild type; Tg, transgenic; LRP-1, lipoprotein receptor-related protein 1; RAGE, receptor for advanced glycation end products; p-gp, p-glycoprotein.

A

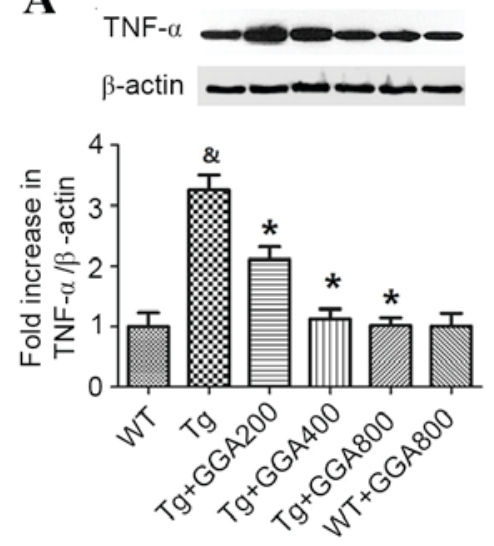

B
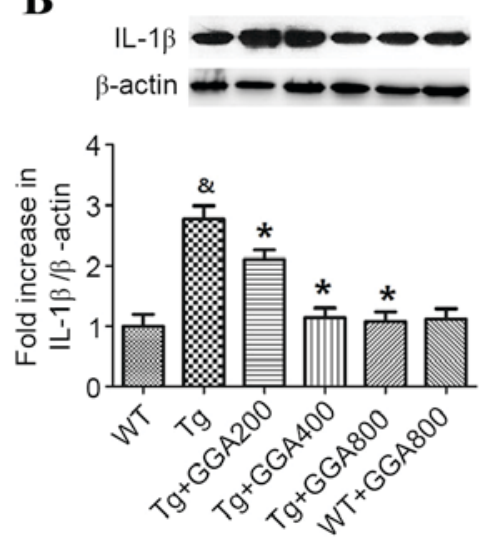

C
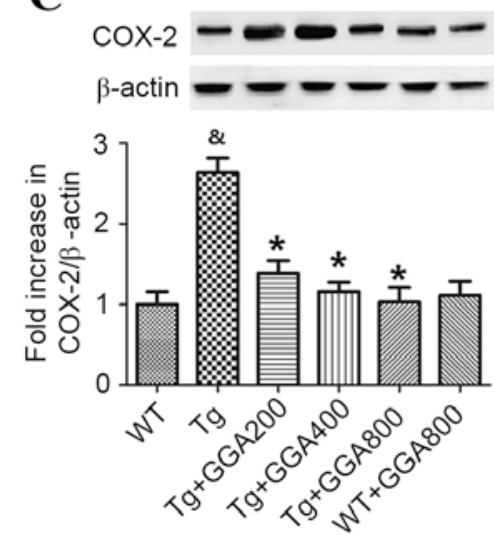

Figure 4. Effect of orally administered GGA on cytokine expression in APP/PS1 mice. Whole cell extracts were prepared from the brains of GGA-treated WT and APP/PS1 mice prior to western blot analysis to measure the levels of (A) TNF- $\alpha,(\mathrm{B})$ IL-1 $\beta$ and (C) COX-2 expression. $\beta$-actin was used as an internal standard. ${ }^{\&} \mathrm{P}<0.05$ vs. untreated WT mice, ${ }^{*} \mathrm{P}<0.05$ vs. untreated Tg mice. GGA, geranylgeranylacetone; APP/PS1, transgenic mouse strain; WT, wild type; Tg, transgenic; TNF- $\alpha$, tumor necrosis factor- $\alpha$; IL-1 $\beta$, interleukin-1 $\beta$; COX-2, cyclooxygenase-2.

GGA affects MAPK signaling in APP/PS1 mice. As presented in Fig. 5A, it was observed by western blot analysis that the levels of phosphorylated (p)c-Jun N-terminal kinase (JNK) did not differ significantly between all groups. By contrast, the levels of p-p38 mitogen-activated protein kinase (p38 MAPK) and p-extracellular signal-regulated kinases (ERK) were significantly higher in $\mathrm{Tg}+\mathrm{GGA} 400$ mice relative to $\mathrm{Tg}$ mice $(\mathrm{P}<0.05)$. In turn, p-p38 and p-ERK levels were significantly decreased in $\mathrm{Tg}+\mathrm{GGA}+$ quercetin mice, compared with Tg+GGA400 mice $(\mathrm{P}<0.05$; Fig. 5B and C). These data suggest a potential role of ERK/p38 MAPK signaling in APP/PS1 mice administered with GGA.

To verify a potential link between ERK/p38 MAPK signaling and the alleviation of AD-related phenotypes in APP/PS1 mice administered with GGA, the effects of ERK/p38 MAPK inhibition on levels of soluble A $\beta$ and LRP-1 were evaluated. In accordance with aforementioned results (Fig. 2), $A \beta 40$ and $A \beta 42$ levels were significantly higher in APP/PS1 mice, relative to WT mice $(\mathrm{P}<0.05)$, and oral administration of GGA significantly reversed the elevated levels of
$\mathrm{A} \beta 40$ and $\mathrm{A} \beta 42$ in $\mathrm{APP} / \mathrm{PS} 1$ mice $(\mathrm{P}<0.05$; Fig. 6A and $\mathrm{B})$. In addition, APP/PS1 mice orally administered with GGA exhibited significant increases in the levels of $A \beta 40$ and $A \beta 42$ following treatment with quercetin, SB-203580 or PD98059 $(\mathrm{P}<0.05$; Fig. 6A and $\mathrm{B})$.

Furthermore, analogous to previous results (Fig. 3A), the level of LRP-1 was significantly lower in the brain of APP/PS1 mice relative to WT mice $(\mathrm{P}<0.05)$, and orally administered GGA significantly increased the lower levels of LRP-1 in APP/PS1 mice $(\mathrm{P}<0.05)$. As depicted in Fig. 6C, it was also observed that treatment with quercetin, SB-203580 or PD98059 following GGA administration in APP/PS1 mice led to a significant decrease in the level of LRP-1, relative to Tg+GGA mice $(\mathrm{P}<0.05)$.

\section{Discussion}

Overexpression of HSP70 alleviates symptoms in a number of neurodegenerative diseases, including Alzheimer's disease, Parkinson's disease, Huntington's disease and spinal 
A
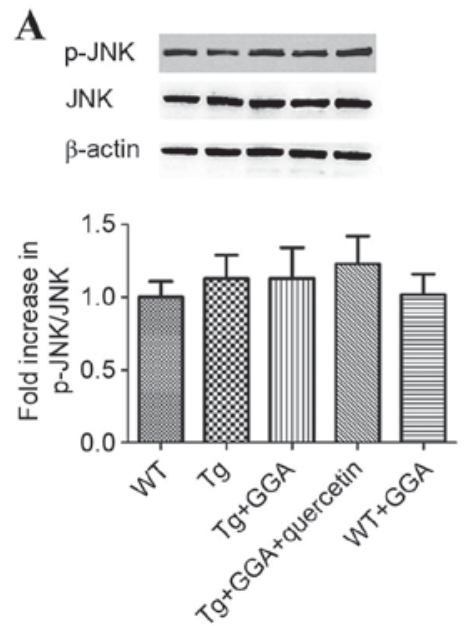

B
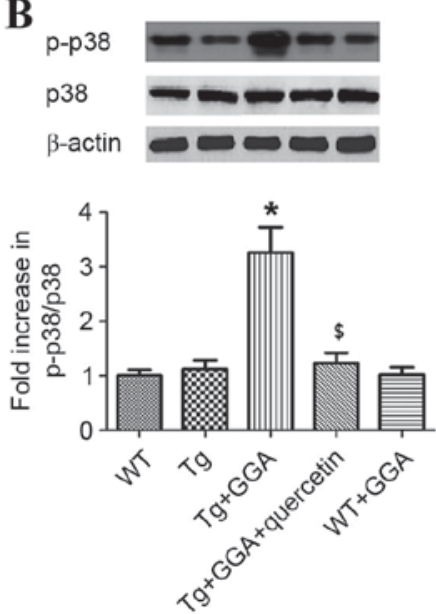

C
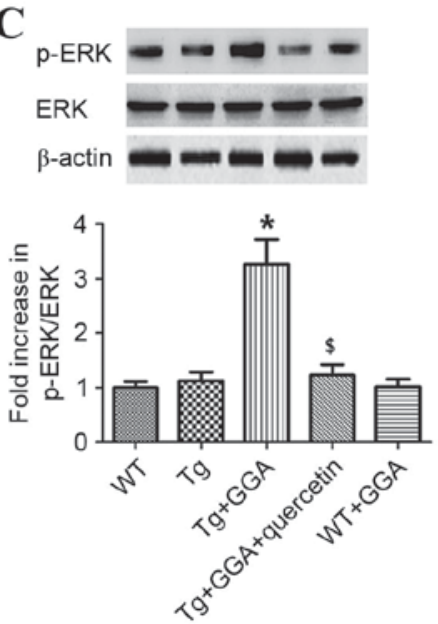

Figure 5. Effects of orally administered GGA on ERK/p38 MAPK signaling in APP/PS1 mice. Whole cell extracts were prepared from the brains of GGA-treated WT and APP/PS1 mice prior to western blot analysis to measure the levels of (A) p-JNK, (B) p-p38 and (C) p-ERK. $\beta$-actin was used as an internal standard. ${ }^{*} \mathrm{P}<0.05$ vs. untreated Tg mice, ${ }^{\$} \mathrm{P}<0.05$ vs. Tg+GGA mice. GGA, geranylgeranylacetone; ERK, extracellular signal-regulated protein kinases; $\mathrm{p} 38 \mathrm{MAPK}$, p38 mitogen-activated protein kinase; APP/PS1, transgenic mouse strain; WT, wild type; Tg, transgenic; JNK, c-Jun N-terminal kinase; p-, phosphorylated.
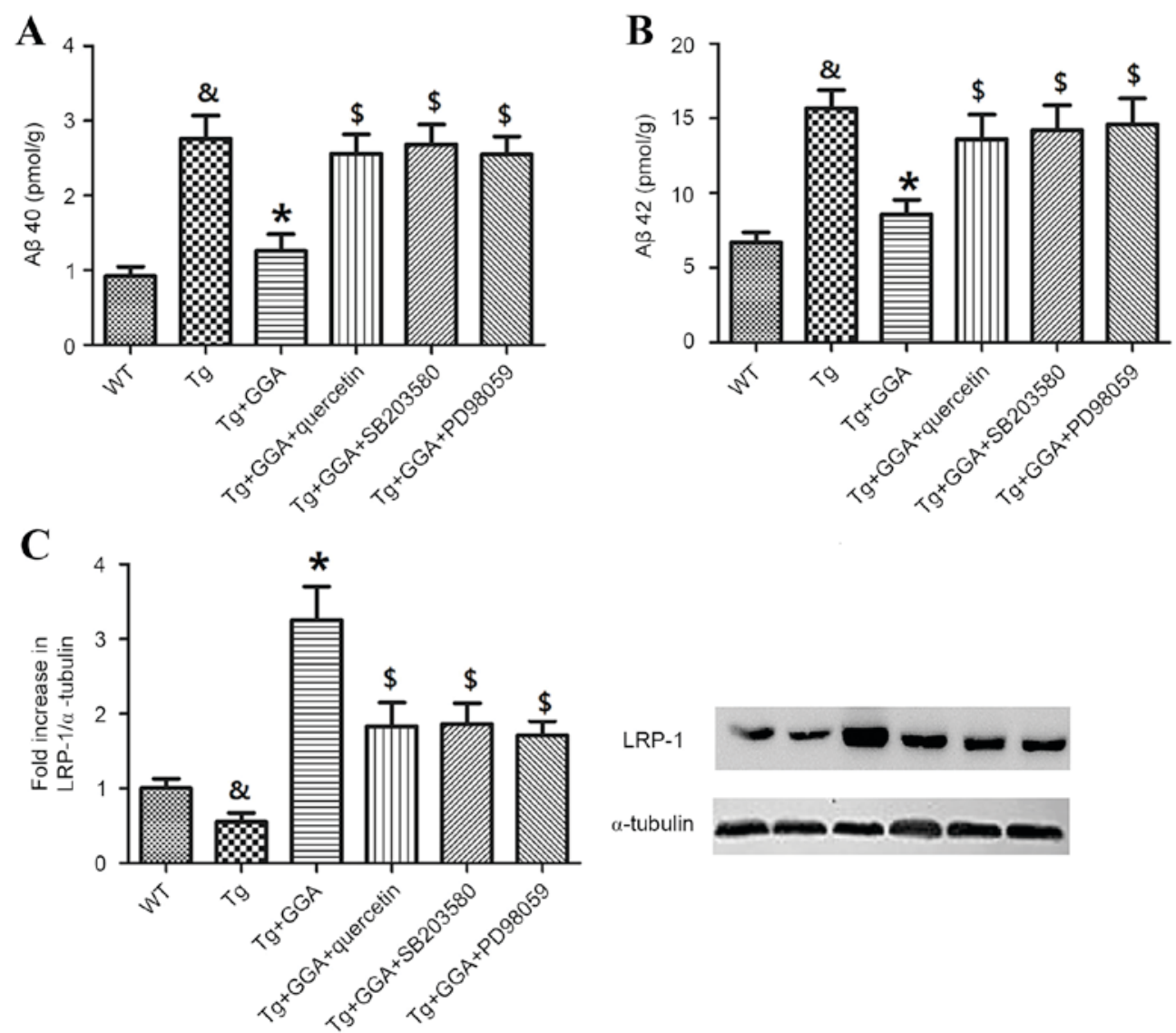

LRP-1

$\alpha$-tubulin

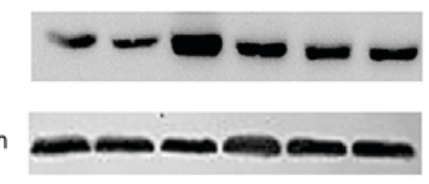

Figure 6. Effects of ERK/p38 MAPK inhibition on pathological phenotypes in APP/PS1 mice. Following oral administration with GGA, WT and APP/PS1 mice were treated with quercetin, SB-203580 or PD98059 to evaluate the involvement of ERK/p38 MAPK signaling in the alleviation of pathological phenotypes in GGA-treated APP/PS1 mice. Levels of soluble (A) A 340 and (B) A 342 in the brain of WT and APP/PS1 mice were measured by sandwich ELISA. (C) The level of LRP-1 expression was measured by western blotting. ${ }^{\circledR} \mathrm{P}<0.05$ vs. untreated WT mice, ${ }^{*} \mathrm{P}<0.05$ vs. untreated Tg mice, ${ }^{\mathrm{S}} \mathrm{P}<0.05$ vs. Tg+GGA mice. ERK, extracellular signal-regulated protein kinases; p38 MAPK, p38 mitogen-activated protein kinase; APP/PS1, transgenic mouse strain; GGA, geranylgeranylacetone; SB-203580, p38 inhibitor; PD98059, ERK inhibitor; A $\beta$, amyloid- $\beta$; LRP-1, lipoprotein receptor-related protein 1; WT, wild type; Tg, transgenic.

cerebellar ataxias $(19,28)$, and thus HSP70 inducers may be potential therapeutics in the treatment of neurodegeneration. It has been demonstrated in a mouse model of $\mathrm{AD}$ that oral administration of GGA alleviates AD-related phenotypes (29), and in the present study, the effects of GGA on the phenotypes displayed by a transgenic APP/PS1 mouse model of AD were 
evaluated. It has also been previously documented that GGA exerts antiulcer effects on gastric ulcers following its absorption through the intestinal mucosa (30), and that GGA is able to pass through the BBB (31), suggesting that HSP70 expression may be influenced by orally administered GGA. In the present study, it was observed that oral administration of GGA rescued cognitive impairment and reversed the pathological phenotypes (decreased levels of A $\beta 40$ and A $\beta 42$ ) in APP/PS1 mice. These results are analogous to those observed in APP23 mice administered with GGA (29), suggesting that GGA has an alleviative effect on AD-related phenotypes in the brain of APP/PS1 mice.

In addition, it was demonstrated that orally administered GGA did not affect the levels of RAGE and p-gp in the brain of APP/PS1 mice, while levels of LRP-1 were significantly increased and levels of TNF- $\alpha$, IL- $1 \beta$ and COX-2 were significantly decreased. Previous studies have demonstrated associations between AD and LRP-1, RAGE and p-gp $(32,33)$. However, the current study observed that levels of RAGE and p-gp in APP/PS1 mice were not affected by orally administered GGA, possibly due to a direct effect of HSP70 upregulation on LRP-1 in APP/PS1 mice. Previous studies have also investigated the role of inflammation in the pathogenesis of AD. Inflammation typically occurs in regions that are pathologically susceptible in AD brains and is accompanied by elevated levels of proinflammatory cytokines, including IL-1 $\beta$, IL-1 and IL-6 (34-36). Considering that LRP-1 serves a key role in $\mathrm{A} \beta$ elimination from the brain (37) and that HSPs have protective effects in inflammation (38), oral administration of GGA may have inhibited inflammation and increased the levels of LRP-1, thus stimulating A $\beta$ clearance, through upregulation of HSP70 in APP/PS1 mice.

In addition to AD-related phenotypes, the present study found that ERK/p38 MAPK signaling was also affected by GGA treatment, with significant increases in levels of p-p38 and p-ERK observed in the brain of GGA-treated APP/PS1 mice. In a previous study by Colombo et al (39), it was demonstrated in an $\mathrm{AD}$ model that $\mathrm{JNK}$ regulated the phosphorylation and degradation of amyloid precursor protein (APP), thus causing significant decreases in the levels of APP, A $\beta$ oligomers and $A \beta$ fragments, thus suggesting a link between the JNK pathway and APP metabolism. By contrast, the present study found that the levels of p-JNK did not differ significantly in GGA-treated APP/PS1 mice relative to WT and Tg mice. This may be due to differences between the current mouse model and the H4-APP ${ }^{\mathrm{sw}}$ cell line employed by Colombo et al (39).

The ERK/p38 MAPK pathway also regulates neuroinflammation, neuronal differentiation and synaptic plasticity (40-42), and p38 MAPK is considered to be a novel therapeutic target in the treatment of AD (43). Furthermore, a study by Khan and Alkon (44) identified AD-specific alterations in the ratio of p-Erk1/Erk2. In the current study, the role of ERK/p38 MAPK signaling in GGA-treated APP/PS1 mice was evaluated by subsequent treatment with either SB-203580 (p38 inhibitor) or PD98059 (ERK inhibitor). Significantly increased levels of soluble $A \beta 40$ and $A \beta 42$ in GGA-treated APP/PS1 mice were detected following SB-203580 or PD98059 administration. A significant decrease in the level of LRP-1 was also observed in GGA-treated APP/PS1 mice following SB-203580 or PD98059 administration. These results indicate that orally administered GGA in APP/PS1 mice suppresses AD-related phenotypes through regulation of the ERK/p38 MAPK signaling pathway. In conclusion, results of the present study suggest a novel molecular mechanism for the suppression of AD-related phenotypes in APP/PS1 mice orally administered with GGA. However, the direct association between LRP-1 and $A \beta$ remains unclear, and requires further study.

\section{References}

1. Katzman R: The prevalence and malignancy of Alzheimer disease: A Major Killer. Arch Neurol 33: 217-218, 2008.

2. Brookmeyer R, Johnson E, Ziegler-Graham K and Arrighi HM: Forecasting the global burden of Alzheimer's disease. Alzheimers Dement 3: 186-191, 2007.

3. McKhann G, Drachman D, Folstein M, Katzman R, Price D and Stadlan EM: Clinical diagnosis of Alzheimer's disease: Report of the NINCDS-ADRDA Work Group under the auspices of Department of Health and Human Services Task Force on Alzheimer's Disease. Neurology 34: 939-944, 1984.

4. Hardy J and Selkoe DJ: The amyloid hypothesis of Alzheimer's disease: Progress and problems on the road to therapeutics. Science 297: 353-356, 2002.

5. Whitwell JL, Dickson DW, Murray ME, Weigand SD, Tosakulwong N, Senjem ML, Knopman DS, Boeve BF, Parisi JE, Petersen RC, et al: Neuroimaging correlates of pathologically defined subtypes of Alzheimer's disease: A case-control study. Lancet Neurol 11: 868-877, 2012

6. Kurz A and Perneczky R: Amyloid clearance as a treatment target against Alzheimer's disease. J Alzheimers Dis 24 (Suppl 2): S61-S73, 2011.

7. Haass C and Selkoe DJ: Soluble protein oligomers in neurodegeneration: Lessons from the Alzheimer's amyloid beta-peptide. Nat Rev Mol Cell Biol 8: 101-112, 2007.

8. Tanzi RE, Moir RD and Wagner SL: Clearance of Alzheimer's A $\beta$ Peptide: The Many Roads to Perdition. Neuron 43: 605-608, 2004.

9. Yoon SS and Jo SA: Mechanisms of amyloid- $\beta$ peptide clearance: Potential therapeutic targets for Alzheimer's disease. Biomol Ther (Seoul) 20: 245-255, 2012.

10. Zlokovic BV: Clearing amyloid through the blood-brain barrier. J Neurochem 89: 807-811, 2004.

11. Tuppo EE and Arias HR: The role of inflammation in Alzheimer's disease. Int J Biochem Cell Biol 37: 289-305, 2015.

12. Selkoe DJ: Cell biology of protein misfolding: The examples of Alzheimer's and Parkinson's diseases. Nat Cell Biol 6: 1054-1061, 2004.

13. Forloni G, Terreni L, Fogliarino S, Invernizzi R, Assini A, Ribizzi G, Negro A, Calabrese E, Volonté MA, Mariani C, et al: Protein misfolding in Alzheimer's and Parkinson's disease: Genetics and molecular mechanisms. Neurobiol Aging 23: 957-976, 2002.

14. Liberek K, Lewandowska A and Zietkiewicz S: Chaperones in control of protein disaggregation. EMBO J 27: 328-335, 2008.

15. Meriin $A B$ and Sherman MY: Role of molecular chaperones in neurodegenerative disorders. Int J Hyperthermia 21: 403-419, 2009.

16. Wyttenbach A and Arrigo AP: The role of heat shock proteins during neurodegeneration in Alzheimer's, Parkinson's and Huntington's Disease. Heat Shock Proteins in Neural Cells: 81-99, 2009.

17. Bobkova NV, Garbuz DG, Nesterova I, Medvinskaya N, Samokhin A, Alexandrova I, Yashin V, Karpov V, Kukharsky MS, Ninkina NN, et al: Therapeutic effect of exogenous hsp70 in mouse models of Alzheimer's disease. J Alzheimers Dis 38: 425-435, 2014.

18. Sinadinos C, Quraishe S, Sealey M, Samson PB, Mudher A and Wyttenbach A: Low endogenous and chemical induced heat shock protein induction in a 0 N3Rtau-expressing drosophila larval model of Alzheimer's disease. J Alzheimers Dis 33: 1117-1133, 2013.

19. Hoshino T, Murao N, Namba T, Takehara M, Adachi H, Katsuno M, Sobue G, Matsushima T, Suzuki T and Mizushima T: Suppression of Alzheimer's disease-related phenotypes by expression of heat shock protein 70 in mice. J Neurosci 31: $5225-5234,2011$ 
20. Mikuriya T, Sugahara K, Takemoto T, Tanaka K, Takeno K, Shimogori H, Nakai A and Yamashita H: Geranylgeranylacetone, a heat shock protein inducer, prevents acoustic injury in the guinea pig. Brain Res 1065: 107-114, 2005.

21. Jankowsky JL, Fadale DJ, Anderson J, Xu GM, Gonzales V, Jenkins NA, Copeland NG, Lee MK, Younkin LH, Wagner SL, et al: Mutant presenilins specifically elevate the levels of the 42 residue beta-amyloid peptide in vivo: Evidence for augmentation of a 42 -specific gamma secretase. Hum Mol Genet 13: 159-170, 2004.

22. Katsuno M, Sang CH, Adachi H, Minamiyama M, Waza M, Tanaka F, Doyu M and Sobue G: Pharmacological induction of heat-shock proteins alleviates polyglutamine-mediated motor neuron disease. Proc Natl Acad Sci USA 102: 16801-16806, 2005.

23. Bromley-Brits K, Deng Y and Song W: Morris water maze test for learning and memory deficits in Alzheimer's disease model mice. J Vis Exp: pii: e2920, 2011.

24. Hughes RN: The value of spontaneous alternation behavior $(\mathrm{SAB})$ as a test of retention in pharmacological investigations of memory. Neurosci Biobehav Rev 28: 497-505, 2004.

25. Broadbent NJ, Gaskin S, Squire LR and Clark RE: Object recognition memory and the rodent hippocampus. Learn Mem 17: $5-11,2009$.

26. Iwata N, Mizukami H, Shirotani K, Takaki Y, Muramatsu S, Lu B, Gerard NP, Gerard C, Ozawa K and Saido TC: Presynaptic localization of neprilysin contributes to efficient clearance of amyloid-beta peptide in mouse brain. J Neurosci 24: 991-998, 2004.

27. Munoz L and Ammit AJ: Targeting p38 MAPK pathway for the treatment of Alzheimer's disease. Neuropharmacology 58: $561-568,2010$.

28. Adachi H, Katsuno M, Waz M, Minamiyam M, Tanak F and Sobue G: Heat shock proteins in neurodegenerative diseases: Pathogenic roles and therapeutic implications. Int J Hyperthermia 25: 647-654, 2009.

29. Hoshino T, Suzuki K, Matsushima T, Yamakawa N, Suzuki T and Mizushima T: Suppression of Alzheimer's disease-related phenotypes by geranylgeranylacetone in mice. PLoS One 8 : e76306, 2013

30. Seno K, Joh T, Yokoyama Y and Itoh M: Role of mucus in gastric mucosal injury induced by local ischemia/reperfusion. J Lab Clin Med 126: 287-293, 1995.

31. Murakami M, Oketani K, Fujisaki H, Wakabayashi T and Ohgo T: Antiulcer effect of geranylgeranylacetone, a new acyclic polyisoprenoid on experimentally induced gastric and duodenal ulcers in rats. Arzneimittelforschung 31: 799-804, 1981.
32. Donahue JE, Flaherty SL, Johanson CE, Duncan JA III, Silverberg GD, Miller MC, Tavares R, Yang W, Wu Q, Sabo E, et al: RAGE, LRP-1, and amyloid-beta protein in Alzheimer's disease. Acta Neuropathol 112: 405-415, 2006.

33. Weller RO, Subash M, Preston SD, Mazanti I and Carare RO: SYMPOSIUM: Clearance of A $\beta$ from the brain in Alzheimer's disease: Perivascular drainage of amyloid- $\beta$ peptides from the brain and its failure in cerebral amyloid angiopathy and Alzheimer's disease. Brain Pathol 18: 253-266, 2008.

34. Finch CE and Morgan TE: Systemic inflammation, infection, ApoE alleles, and Alzheimer disease: A position paper. Curr Alzheimer Res 4: 185-189, 2007.

35. Griffin WS and Mrak RE: Interleukin-1 in the genesis and progression of and risk for development of neuronal degeneration in Alzheimer's disease. J Leukoc Biol 72: 233-238, 2002.

36. Bona DD, Plaia A, Vasto S, Cavallone L, Lescai F, Franceschi C, Licastro F, Colonna-Romano G, Lio D, Candore G and Caruso C: Association between the interleukin-1beta polymorphisms and Alzheimer's disease: A systematic review and meta-analysis. Brain Res Rev 59: 155-163, 2008.

37. Deane R, Sagare A and Zlokovic BV: The role of the cell Surface LRP and Soluble LRP in blood-brain barrier abeta clearance in Alzheimer's disease. Curr Pharm Des 14: 1601-1605, 2008.

38. Jacquier-Sarlin MR, Fuller K, Dinh-Xuan AT, Richard MJ and Polla BS: Protective effects of the hsp70 in inflammation. Experimentia 50: 1031-1038, 1994.

39. Colombo A, Bastone A, Ploia C, Sclip A, Salmona M, Forloni G and Borsello T: JNK regulates APP cleavage and degradation in a model of Alzheimer's disease. Neurobiol Dis 33: 518-525, 2013.

40. Webber KM, Smith MA, Lee HG, Harris PL, Moreira P, Perry G and Zhu X: Mitogen- and stress-activated protein kinase 1: Convergence of the ERK and p38 pathways in Alzheimer's disease. J Neurosci Res 79: 554-560, 2005.

41. Roux PP and Blenis J: ERK and p38 MAPK-activated protein kinases: A family of protein kinases with diverse biological functions. Microbiol Mol Biol Rev 68: 320-344, 2004.

42. Sarina, Yagi Y, Nakano O, Hashimoto T, Kimura K, Asakawa Y, Zhong M, Narimatsu S and Gohda E: Induction of neurite outgrowth in PC12 cells by artemisinin through activation of ERK and p38 MAPK signaling pathways. Brain Res 1490: 61-71, 2013.

43. Lenka M and Alaina JA: Targeting p38 MAPK pathway for the treatment of Alzheimer's disease. Neuropharmacology 58: $561-568,2010$.

44. Khan TK and Alkon DL: Alzheimer's disease-specific alterations of the Erk1/Erk2 phosphorylation ratio. Journal, 2009. 